\title{
Septal Deviation Is Associated with Maxillary Sinus Fungus Ball in Male Patients
}

\author{
Hidetoshi Oshima, ${ }^{1}$ Kazuhiro Nomura, ${ }^{1,2}$ Mitsuru Sugawara, ${ }^{2}$ Kazuya Arakawa, ${ }^{2}$ \\ Takeshi Oshima ${ }^{1}$ and Yukio Katori ${ }^{1}$ \\ ${ }^{1}$ Department of Otolaryngology-Head and Neck Surgery, Tohoku University Graduate School of Medicine, \\ Sendai, Miyagi, Japan \\ ${ }^{2}$ Department of Otolaryngology, Tohoku Kosai Hospital, Sendai, Miyagi, Japan
}

\begin{abstract}
Fungus is one of the causes of chronic rhinosinusitis. If the fungus occupies the sinus but does not invade the sinonasal mucosa, this is called sinus fungus ball. Any association between anatomical variations and fungus ball remains unclear. Sinus fungus ball is defined as non-invasive chronic fungal rhinosinusitis occurring in immunocompetent patients, and the maxillary sinus is the most commonly affected. The etiology of maxillary sinus fungus ball remains unclear. This study assessed the potential contribution of anatomical variations, such as deviated nasal septum, concha bullosa, and Haller cell to the development of fungus ball in the maxillary sinus. Concha bullosa and Haller cell are structural variations that narrow the nasal airflow passage and contribute to chronic rhinosinusitis. The involvement of these variations has been investigated in chronic sinusitis but not in sinus fungus ball. Preoperative computed tomography findings of 103 patients with maxillary sinus fungus ball were evaluated retrospectively. Septal deviation and Haller cell were not correlated with the side of maxillary sinus fungus ball. Concha bullosa was more common on the unaffected side $(p=0.099)$. When we analyzed males and females separately, maxillary sinus fungus ball was more common on the concave side of the deviated septum in only male patients $(p=$ 0.006). The high incidence of maxillary fungus ball in the concave side may reflect the consequences of the traumatic effects caused by wall shear stress of the high-velocity airflow and the increased chance of inhaling fungus spores.
\end{abstract}

Keywords: concha bullosa; fungus ball; Haller cell; non-invasive fungal sinusitis; septal deviation Tohoku J. Exp. Med., 2014 March, 232 (3), 201-206. C 2014 Tohoku University Medical Press

\section{Introduction}

Chronic rhinosinusitis is defined as a group of disorders characterized by inflammation of the mucosa of the nose and paranasal sinuses of at least 12 weeks duration. Fungus is one of the causes of chronic rhinosinusitis. Fungal rhinosinusitis is encountered in about $10 \%$ of patients requiring surgery for complaints of the nose and sinuses, and fungal or mixed fungal and bacterial infections account for $13.5 \%$ to $28.5 \%$ of all cases of maxillary sinusitis (Grosjean and Weber 2007). Fungal sinusitis may be non-invasive and invasive (deShazo et al. 1997; Schubert 2001; Grosjean and Weber 2007). Non-invasive fungal sinusitis occurs in immunocompetent patients and is characterized by the absence of fungal hyphae in the mucosa of the sinus. This type is subdivided into fungus ball and allergic fungal sinusitis. The diagnosis of allergic fungal sinusitis is based on the presence of inspissated allergic mucin that is grossly evident at surgery and histologically positive for fungal hyphae (Schubert 2009). Sinus fungus ball is a form of fungal sinusitis that is defined as non-invasive chronic fungal sinusitis without inspissated allergic mucin. Fungus ball most commonly occurs in the maxillary sinus, followed by the sphenoid sinus (Grosjean and Weber 2007). Fungus ball of the paranasal sinuses is mostly encountered in older individuals (Dufour et al. 2006; Nicolai et al. 2009; Lai et al. 2011). Female predominance is reported in most series (Klossek et al. 1997; Dufour et al. 2006; Nicolai et al. 2009; Lai et al. 2011).

The pathogenesis of fungus ball remains unclear, but two routes of entry have been suggested (Grosjean and Weber 2007): the so-called "aerogenic" pathway in which high quantities of airborne fungus spores enter the sinus through the natural ostium, and the odontogenic "iatrogenic" pathway in which fungal colonization of the maxillary sinus occurs through iatrogenic oroantral communication secondary to dental extraction, periodontal damage, or most often endodontic treatment with overfilling of the den-

Received August 27, 2013; revised and accepted February 25, 2014. Published online March19, 2014; doi: 10.1620/tjem.232.201.

Correspondence: Hidetoshi Oshima, M.D., Department of Otolaryngology-Head and Neck Surgery, Tohoku University Graduate School of Medicine, 1-1 Seiryo-machi, Aoba-ku, Sendai, Miyagi 980-8574, Japan.

e-mail: tohokuoshima-ent@yahoo.co.jp 
tal canal (Odell and Pertl 1995). These two theories are controversial and no definitive evidence has been presented. The aerogenic theory states that inhaled fungus spores deposited in the sinuses (usually the ethmoid sinus) become pathogenic as the sinuses become an anaerobic environment (Nicolai et al. 2009). Fungal sinusitis may be a specific form or complication of chronic recurring sinusitis (Stammberger 1985). The maxillary sinus is the most common sinus involved in adult chronic sinusitis (Slavin 1988). However, osteomeatal complex obstruction is irrelevant to the growth of maxillary sinus fungus ball and contradicts these hypotheses (Tsai et al. 2006). Septal deviation, concha bullosa, and Haller cell are anatomical variations that contribute to narrowing of the drainage pathway of the maxillary sinus. Concha bullosa is a pneumatized cavity within the middle turbinate. Haller cell is a type of extramural ethmoidal cells that extend into the inferomedial orbital floor. The involvement of these variations has been investigated in chronic sinusitis but not in sinus fungus ball (Bolger et al. 1991; Laine and Smoker 1992; Sivasli et al. 2003; Caughey et al. 2005; Al-Qudah 2008; Orlandi 2010). Bacteria and fungi have different sizes and structures, and provoke different reactions in the human body. If the maxillary sinus is wide open and well ventilated, more airborne fungus spores can enter the sinus. On the other hand, if the sinus has poor ventilation, spores can easily persist after entry. Therefore, whether tendency to form fungal ball has any relationship to good or poor ventilation of the maxillary sinus is an unknown and interesting issue.

Recently, two studies have investigated the side of the fungus ball and the anatomical structures. Analysis of the incidence of septal deviation and concha bullosa in 52 cases of maxillary and sphenoid fungus balls found no correlation between the anatomical structure and the side of the fungus ball (Tsai et al. 2012). However, the drainage pathways of the maxillary and sphenoid sinuses are anatomically separated and concha bullosa does not disturb the passage to the sphenoid sinus (Nomura et al. 2013a). The present study focused on maxillary sinus fungus ball. Soft tissue volume, which is affected by inflammation, has been suggested as an anatomical factor of the occurrence of fungal ball based on computed tomography (CT) measurements (Hwang et al. 2012). However, soft tissue volume increases with inflammation after the occurrence of fungus disease. Therefore, the volume of middle meatus and nasal valve pre- and post-infection differs. We assessed septal deviation, concha bullosa, and Haller cell. These structures consist of bone, which is not affected by the occurrence of fungal sinusitis. The characteristics of fungal infection differ in various regions across the globe. A study of the composition of ambient air showed different fungus species present in France and the USA (Dufour et al. 2005). This study analyzed cases of sinus fungus ball in the Japanese population.

The present study analyzed the relationships between the occurrence of maxillary sinus fungus ball and the presence of various anatomical risk factors using multivariate logistic regression analysis.

\section{Materials and Methods}

This study evaluated patients with maxillary sinus fungus ball who underwent endoscopic sinus surgery at Tohoku University Hospital, Sendai, Japan, between January 2003 and July 2013 and at Tohoku Kosai Hospital, Sendai, Japan, between March 2010 and March 2013. The inclusion criteria were surgically confirmed maxillary sinus fungus ball and $\mathrm{CT}$ scans acquired prior to surgery. The exclusion criteria were previous surgery on the sinonasal area, fungus ball in other sinuses, and fungus balls in the bilateral maxillary sinuses. The CT scans of 103 patients were reviewed and the anatomical variations were measured and classified.

The anatomical variations examined were septal deviation and direction, and presence of concha bullosa or Haller cell. Septal deviation of $10^{\circ}$ or more was considered to be a separate factor (Fig. 1). Septal deviation angle was defined as the angle between a line drawn
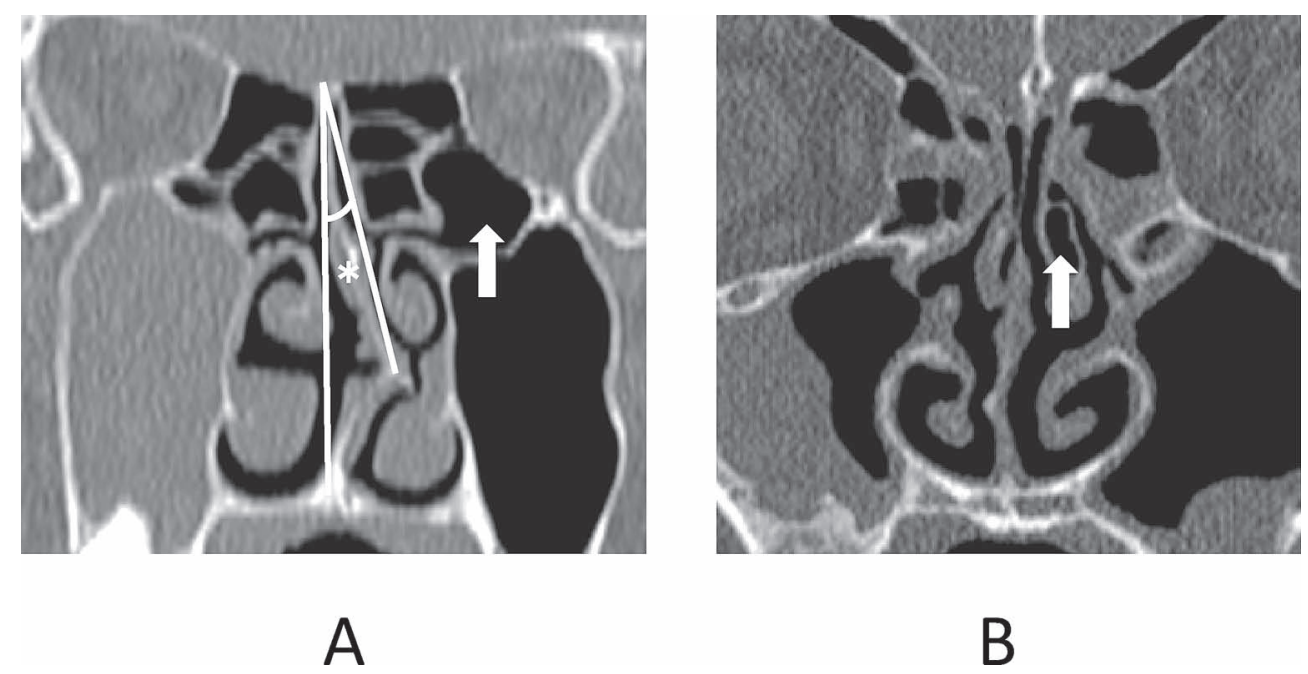

Fig. 1. Examples of anatomical variations.

(A) Septal deviation and Haller cell (arrow). Asterisk shows septal deviation angle. (B) Concha bullosa (arrow). 
from the superior insertion of the septum at the crista galli to the inferior insertion of the septum at the level maxillary crest and a line drawn from the superior insertion of the septum at the crista galli to the apex of the septal deviation (Harar et al. 2004). A dummy variable was set equal to 1 if the septal deviation was directed to the side of concern, and 0 otherwise. The relationships between the occurrence of maxillary sinus fungus ball and the anatomical risk factors were analyzed using multivariate logistic regression analysis with Stata/MP 11.1 for Mac (StataCorp LP, College Station, TX). The study was approved by the institutional review board of Tohoku University Graduate School of Medicine.

\section{Results}

The pathological findings in this study are summarized in Table 1. Positive identification was achieved in 90 histological specimens. The causative fungus was mainly

Table 1. Histological examination of fungus ball.

\begin{tabular}{lr}
\hline Total no. of patients & 103 \\
Fungus & 90 \\
Aspergillus & 87 \\
Actinomycosis & 2 \\
Candida & 1 \\
Fungus not identified & 13 \\
\hline
\end{tabular}

The causative fungus was mainly Aspergillus species $(n=87 / 90,96.7 \%)$.
Aspergillus species $(n=87 / 90,96.7 \%)$. The 70 female $(68 \%)$ and 33 male (32\%) patients were aged from 33 to 89 years (mean 66.4 years). The analysis of anatomical variations is summarized in Table 2. Maxillary fungus ball was found in 22 cases and intact maxillary sinus in 27 cases within the convex side. Even if limited to only cases with severe deviation $\left(\geq 10^{\circ}\right)$, the affected side was the convex side in 9 cases and the unaffected side in 9 cases. Concha bullosa was more common on the unaffected side, but was not statistically significant $(p=0.099)$. Similarly, Haller cell was found in both affected and unaffected sides. None of the anatomical variations were significantly correlated to the occurrence of maxillary sinus fungus ball (Table 3 ). When we analyzed males and females separately, maxillary fungus ball was more common on the concave side of the septal deviation in male patients $(p=0.006)$. Such an association was not observed in female cases $(p=0.842)$ (Table 4). Concha bullosa and Haller cell were not associated with the side of the maxillary fungus ball in both sexes.

\section{Discussion}

The detection rate of fungal hyphae in fungus ball has been relatively high $[n=102 / 109,93.6 \%$ (Klossek et al. 1997), and $n=151 / 164,92.1 \%$ (Dufour et al. 2005)]. Histological examination of the specimens demonstrated a large amount of fungal hyphae in all cases, but histological analysis identified the most prevalent Aspergillus species in

Table 2. Prevalence of anatomical variations in sides affected and unaffected by maxillary sinus fungus ball.

\begin{tabular}{ccc}
\hline \multicolumn{1}{c}{ Abnormality } & Affected side & Unaffected side \\
\hline $\begin{array}{c}\text { Nasal septum deviated to } \\
\text { (male, female) }\end{array}$ & 22 & 27 \\
Nasal septum severely deviated to $\left(\geq 10^{\circ}\right)$ & $(6,16)$ & $(13,14)$ \\
$\quad($ male, female) & 9 & 9 \\
Concha bullosa & $(4,5)$ & $(4,5)$ \\
$\quad($ male, female $)$ & 13 & 21 \\
Haller cell & $(3,10)$ & $(7,14)$ \\
(male, female) & 24 & 22 \\
\hline
\end{tabular}

CT detection of anatomical variations $(n=103)$. Nasal septum was deviated to affected side in 22 cases and to unaffected side in 27 cases. When limited to cases with severe deviation $\left(\geq 10^{\circ}\right)$, the fungus ball was seen in the same number of cases in both affected and unaffected sides. Concha bullosa (pneumatized middle turbinate) was more common on the unaffected side, but was not statistically significant $(p=0.099)$. Haller cell was found in both affected and unaffected sides.

Table 3. Multivariate analysis of factors associated with maxillary sinus fungus ball.

\begin{tabular}{lcccc}
\hline & Adjusted OR $(95 \% \mathrm{CI})$ & $P$ value & Unadjusted OR $(95 \% \mathrm{CI})$ & $P$ value \\
\hline Obstruction due to septal deviation & $0.639(0.290-1.405)$ & 0.265 & $0.765(0.401-1.456)$ & 0.414 \\
Obstruction due to severe septal deviation $\left(\geq 10^{\circ}\right)$ & $1.485(0.453-4.871)$ & 0.515 & $1.000(0.380-2.630)$ & 1.000 \\
Concha bullosa & $0.524(0.243-1.128)$ & 0.099 & $0.564(0.265-1.198)$ & 0.136 \\
Haller cell & $1.173(0.598-2.303)$ & 0.642 & $1.119(0.580-2.156)$ & 0.738 \\
\hline
\end{tabular}

OR, odds ratio; $\mathrm{CI}$, confidence interval.

These anatomical variations were not significantly correlated to the occurrence of maxillary sinus fungus ball. 
Table 4. Association between septal deviation and maxillary sinus fungus ball in male and female patients.

\begin{tabular}{lcccc}
\hline & Adjusted OR $(95 \% \mathrm{CI})$ & $P$ Value & Unadjusted OR $(95 \% \mathrm{CI})$ & $P$ Value \\
\hline Male & $0.220(0.074-0.653)$ & 0.006 & $0.462(0.233-0.915)$ & 0.027 \\
Female & $1.068(0.560-2.036)$ & 0.842 & $1.071(0.640-1.793)$ & 0.793 \\
\hline
\end{tabular}

Maxillary fungus ball was more common on the concave side of the septal deviation in male patients $(p=0.006)$. In contrast, there was no correlation in female patients $(p=0.842)$.

only $21 / 160$ (13.1\%) samples (Nicolai et al. 2009). In this study, dark brown, cheesy friable materials were confirmed in all cases during surgery. The relatively low identification rate in our study (87.3\%) may be a consequence of necrosis and inflammation. Regional differences in fungi are the other possible explanation (Dufour et al. 2005). In this study, the causative fungus was mainly Aspergillus species ( $n=87 / 90,96.7 \%$ ), as also found by previous studies (Dufour et al. 2005; Nicolai et al. 2009). The clinical characteristics of the patients in this study were similar to those in previously reported series (Klossek et al. 1997; Dufour et al. 2006; Nicolai et al. 2009; Lai et al. 2011). The average age was 66.4 years and female predominance was seen $(68 \%)$.

The present study investigated septal deviation, concha bullosa, and Haller cell as anatomical variations that cause narrowing of the drainage pathway of the maxillary sinus, which decrease the possibility that fungus spores can reach the sinuses, but disturb the drainage pathway, which increases the possibility of spore retention.

Concha bullosa is a pneumatized middle turbinate, which is a possible etiological factor in recurrent sinusitis due to the postulated negative influence on paranasal sinus ventilation and mucociliary clearance in the middle meatus region (Bolger et al. 1991). The prevalence ranges from $8 \%$ to $80 \%$, although $80 \%$ of cases were found in a patient population with chronic sinusitis at ethmoidectomy (Bolger et al. 1991). The pathogenicity of concha bullosa in the etiology of maxillary sinusitis is controversial. A review of 6 studies concluded that involvement of concha bullosa in initiating or sustaining paranasal sinus disease is unlikely to be very important (Jones 2002). On the other hand, analysis of $250 \mathrm{CT}$ scans found that concha bullosa was associated with maxillary sinus disease $(p<0.01)$ (Caughey et al. 2005 ). In our study, concha bullosa was present in $13 \%$ of the sides with maxillary sinus fungus ball and $20 \%$ of the other sides. Surprisingly, concha bullosa was more prevalent on the unaffected side. No significant correlation was found between concha bullosa and maxillary sinus fungus ball (Table 3).

Haller cell is an ethmoid cell that projects beyond the limits of the ethmoid into the maxillary sinus and is located along the maxillary sinus roof. Haller cell was named after the 18th century anatomist Albert von Haller. Haller cell blocks maxillary sinus ventilation and drainage, and so may be an etiologic factor in recurrent maxillary sinusitis (Bolger et al. 1991). Coronal CT found that $10 \%$ of patients had this type of anatomy (Kennedy and Zinreich 1988). The incidence of Haller cell was $45.1 \%$ in another study (Bolger et al. 1991). Review of 5 studies found that Haller cell has no involvement in the pathogenesis of maxillary sinusitis (Jones 2002). In contrast, Haller cell was associated with both ethmoid $(p<0.05)$ and maxillary ( $p<$ $0.01)$ mucosal disease (Caughey et al. 2005). In our study, Haller cell was present in $23 \%$ of the sides with maxillary sinus fungus ball and $21 \%$ of the other sides, indicating no significant correlation between Haller cell and maxillary sinus fungus ball (Table 3 ).

Septal deviation is the most well-known anatomical factor that leads to recurrent sinusitis. Many studies on the effect of septal deviation on sinusitis have been reported with conflicting results. Quantitative and qualitative analysis of previously published studies found that increasing angles of septal deviation were associated with increasing prevalence of rhinosinusitis, septal deviation of $\geq 10^{\circ}$ was significantly associated with rhinosinusitis $\left(p=0.0004, \chi^{2}\right.$ analysis), and the clinical effect was modest with an odds ratio of 1.47 (Orlandi 2010). Finally, all studies that examined the laterality of rhinosinusitis associated with septal deviation found that inflammation was bilateral. In our study, septal deviation was present in $48 \%$ of the cases. The septum deviated toward the sides with maxillary sinus fungus ball in $21 \%$ and toward the intact side in $26 \%$. Deviation of $\geq 10^{\circ}$ was found in $17 \%$ of the cases. The septum was severely deviated to the sides with maxillary sinus fungus ball in 9 cases and to the intact sides in 9 cases, indicating no significant correlation between maxillary sinus fungus ball and septal deviation either in general or in severely deviated cases (Fig. 1A, Table 3). Even severely deviated septum had no impact on laterality. Although in general, concha bullosa tends to occur in the concave side, the maxillary sinus ostium is also narrowed (Suzuki et al. 1999), but such a tendency was not observed in our cases (Spearman correlation analysis; concha bullosa and septal deviation, $r=-0.06, p=0.34$; concha bullosa and severe septal deviation, $r=0.05, p=0.50$ ). When males and females were analyzed separately, significant association was observed. Maxillary fungus ball was significantly common on the concave side of the deviated septum in male patients (Table 3). The reason why this association was observed only in males is not clear, but the relatively larger size of the male head might be more sensitive to the airflow of the nasal cavity. The concave side shows increased velocities and turbulence in the middle 
meatal area (Chen et al. 2009). The traumatic effects of the wall shear stress caused by the high velocity of airflow in the concave side might result in the high incidence of maxillary fungus ball. Recently we reported a correlation between septal deviation and sinonasal papilloma (Nomura et al. 2013b). Papilloma was more often seen in the concave side, as found in this study on fungus ball. Mucosal injury due to wall shear stress might increase the chance of infection by human papilloma virus.

This study was focused on anatomical structures. The contralateral side was used as the negative control. Therefore, other risk factors, such as diabetes, smoking, and asthma, which may predispose to fungal sinusitis, can be ignored. Although chronic sinusitis tended to occur in the narrower side, maxillary sinus fungus ball did not show any such tendency. Furthermore, when limited to male cases, maxillary fungus ball was more prevalent in the concave side. Fungal sinusitis occurring in well-ventilated maxillary sinus might be related to the chance of inhaling fungus spores. Fungus ball is more likely to occur in infected or inflammatory mucosa than in normal maxillary sinus (Stammberger 1985). Such vulnerable conditions of the maxillary sinus depend on the size of the natural ostium and mucociliary function in the maxillary sinus mucosa. The size of the natural ostium and the mucociliary function are not measurable by $\mathrm{CT}$, so predicting the risk of sinus fungus ball is difficult.

In conclusion, most well-known anatomical variations that narrow sinonasal airflow, such as septal deviation, concha bullosa, and Haller cell, do not contribute to the occurrence of maxillary sinus fungus ball in contrast to chronic sinusitis. Concha bullosa was more prevalent in the intact side. Maxillary sinus fungus ball was more common on the concave side of the deviated septum in only male patients. The high incidence of maxillary fungus ball in the concave side may reflect the consequences of the traumatic effects caused by wall shear stress of the high-velocity airflow and the increased chance of inhaling fungus spores.

\section{Conflict of Interest}

The authors declare no conflict of interest.

\section{References}

Al-Qudah, M. (2008) The relationship between anatomical variations of the sino-nasal region and chronic sinusitis extension in children. Int. J. Pediatr. Otorhinolaryngol., 72, 817-821.

Bolger, W.E., Butzin, C.A. \& Parsons, D.S. (1991) Paranasal sinus bony anatomic variations and mucosal abnormalities: CT analysis for endoscopic sinus surgery. Laryngoscope, 101, 56-64.

Caughey, R.J., Jameson, M.J., Gross, C.W. \& Han, J.K. (2005) Anatomic risk factors for sinus disease: fact or fiction? $\mathrm{Am}$. J. Rhinol., 19, 334-339.

Chen, X.B., Lee, H.P., Chong, V.F. \& Wang, de Y. (2009) Assessment of septal deviation effects on nasal air flow: a computational fluid dynamics model. Laryngoscope, 119, 1730-1736.

deShazo, R.D., O’Brien, M., Chapin, K., Soto-Aguilar, M., Swain, R., Lyons, M., Bryars, W.C. \& Alsip, S. (1997) Criteria for the diagnosis of sinus mycetoma. J. Allergy Clin. Immunol., 99, 475-485.

Dufour, X., Kauffmann-Lacroix, C., Ferrie, J.C., Goujon, J.M., Rodier, M.H., Karkas, A. \& Klossek, J.M. (2005) Paranasal sinus fungus ball and surgery: a review of 175 cases. Rhino$\log y$, 43, 34-39.

Dufour, X., Kauffmann-Lacroix, C., Ferrie, J.C., Goujon, J.M., Rodier, M.H. \& Klossek, J.M. (2006) Paranasal sinus fungus ball: epidemiology, clinical features and diagnosis. A retrospective analysis of 173 cases from a single medical center in France, 1989-2002. Med. Mycol., 44, 61-67.

Grosjean, P. \& Weber, R. (2007) Fungus balls of the paranasal sinuses: a review. Eur. Arch. Otorhinolaryngol., 264, 461-470.

Harar, R.P.S., Chadha, N.K. \& Rogers, G. (2004) The role of septal deviation in adult chronic rhinosinusitis: a study of 500 patients. Rhinology, 42, 126-130.

Hwang, S.H., Kang, J.M., Cho, J.H. \& Kim, B.G. (2012) What is the relationship between the localization of maxillary fungal balls and intranasal anatomic variations? Clin. Exp. Otorhinolaryngol., 5, 213-217.

Jones, N.S. (2002) CT of the paranasal sinuses: a review of the correlation with clinical, surgical and histopathological findings. Clin. Otolaryngol. Allied Sci., 27, 11-17.

Kennedy, D.W. \& Zinreich, S.J. (1988) The functional endoscopic approach to inflammatory sinus disease: current perspectives and technique modifications. Am. J. Rhinol., 2, 89-96.

Klossek, J.M., Serrano, E., Péloquin, L., Percodani, J., Fontanel, J.P. \& Pessey, J.J. (1997) Functional endoscopic sinus surgery and 109 mycetomas of paranasal sinuses. Laryngoscope, 107, 112-117.

Lai, J.C., Lee, H.S., Chen, M.K. \& Tsai, Y.L. (2011) Patient satisfaction and treatment outcome of fungus ball rhinosinusitis treated by functional endoscopic sinus surgery. Eur. Arch. Otorhinolaryngol., 268, 227-230.

Laine, F.J. \& Smoker, W.R. (1992) The ostiomeatal unit and endoscopic surgery: anatomy, variations, and imaging findings in inflammatory diseases. AJR Am. J. Roentgenol., 159, 849-857.

Nicolai, P., Lombardi, D., Tomenzoli, D., Villaret, A.B., Piccioni, M., Mensi, M. \& Maroldi, R. (2009) Fungus ball of the paranasal sinuses: experience in 160 patients treated with endoscopic surgery. Laryngoscope, 119, 2275-2279.

Nomura, K., Nakayama, T., Asaka, D., Okushi, T., Hama, T., Kobayashi, T. \& Otori, N. (2013a) Laterally attached superior turbinate is associated with opacification of the sphenoid sinus. Auris Nasus Larynx, 40, 194-198.

Nomura, K., Ogawa, T., Sugawara, M., Honkura, Y., Oshima, H., Arakawa, K., Oshima, T. \& Katori, Y. (2013b) Association between septal deviation and sinonasal papilloma. Tohoku J. Exp. Med., 231, 315-319.

Odell, E. \& Pertl, C. (1995) Zinc as a growth factor for Aspergillus sp. and the antifungal effects of root canal sealants. Oral Surg. Oral Med. Oral Pathol. Oral Radiol. Endod., 79, 82-87.

Orlandi, R.R. (2010) A systematic analysis of septal deviation associated with rhinosinusitis. Laryngoscope, 120, 1687 1695.

Schubert, M.S. (2001) Fungal rhinosinusitis: diagnosis and therapy. Curr. Allergy Asthma Rep., 1, 268-276.

Schubert, M.S. (2009) Allergic fungal sinusitis: pathophysiology, diagnosis and management. Med. Mycol., 47 Suppl 1, S324S330.

Sivasli, E., Sirikçi, A., Bayazýt, Y.A., Gümüsburun, E., Erbagci, H., Bayram, M. \& Kanlýkama, M. (2003) Anatomic variations of the paranasal sinus area in pediatric patients with chronic sinusitis. Surg. Radiol. Anat., 24, 400-405.

Slavin, R.G. (1988) Sinusitis in adults. J. Allergy Clin. Immunol., 81, 1028-1032.

Stammberger, H. (1985) Endoscopic surgery for mycotic and chronic recurring sinusitis. Ann. Otol. Rhinol. Laryngol. 
Suppl., 119, 1-11.

Suzuki, H., Yamaguchi, T. \& Furukawa, M. (1999) Rhinologic computed tomographic evaluation in patients with cleft lip and palate. Arch. Otolaryngol. Head Neck Surg., 125, 1000-1004.

Tsai, T.L., Guo, Y.C., Ho, C.Y. \& Lin, C.Z. (2006) The role of ostiomeatal complex obstruction in maxillary fungus ball.
Otolaryngol. Head Neck Surg., 134, 494-498.

Tsai, T.L., Lan, M.Y. \& Ho, C.Y. (2012) There is no structural relationship between nasal septal deviation, concha bullosa, and paranasal sinus fungus balls. Scientific WorldJournal, 2012, 181246. 